\title{
Educación ambiental para la disminución del uso de agroquímicos en la comunidad Balsa en Medio (Ecuador)
}

\author{
Environmental education for the reduction of the use of agrochemicals in the \\ Balsa en Medio community (Ecuador)
}

\author{
Ana María Aveiga Ortizi,a, Hugo Miguel Cobeña Navarrete ${ }^{1, b}$, César Alfredo Pinargote Zambrano ${ }^{1, c}$, \\ Oscar Rafael Tinoco Gómez ${ }^{2, d}$, Francisco Alejandro Alcántara Boza ${ }^{2, e}$
}

Recibido: 28/05/2021 - Aprobado: 16/07/2021 - Publicado: 23/12/2021

\begin{abstract}
RESUMEN
La educación ambiental es una herramienta eficaz para aplicar de técnicas de producción sostenibles. El objetivo de esta investigación fue aplicar estrategias de educación ambiental para la disminución del uso de agroquímicos en la comunidad Balsa en Medio (Ecuador). Mediante escalas de tipo Likert, se realizó un diagnóstico del nivel de conocimiento y comportamiento ambiental de los miembros de la comunidad. Después, se ejecutó el plan de capacitación aplicando estrategias ambientales sobre elaboración y aplicación de compost, bioles, bioinsecticidas y biopreparados con microorganismos eficientes en cultivos estacionales. Finalmente, se evaluó el conocimiento y comportamiento ambiental final de los participantes. Los resultados del diagnóstico revelaron que el $100 \%$ de los participantes presentaron un deficiente conocimiento ambiental; mientras que, el $98 \%$ de participantes mostraron comportamiento ambiental inadecuado. Después de la aplicación de un programa de educación ambiental, se obtuvo que el $21,15 \%$ de participantes mostraron conocimiento ambiental excelente; $61,54 \%$ se ubicaron en la categoría bueno y el $17,31 \%$ restante reveló escaso conocimiento; para el comportamiento ambiental, el 44,23\% de participantes se ubicó en adecuado, en contrate, 55,77\% mostró comportamiento ambiental inadecuado. Los talleres impartidos en la comunidad permitieron aumentar de manera significativa el nivel de conocimiento y comportamiento ambiental.
\end{abstract}

Palabras claves: Estrategias de educación ambiental; conocimiento ambiental; comportamiento ambiental; comunidad rural; escala Likert; plan de capacitación.

\begin{abstract}
Environmental education is an effective tool to apply sustainable production techniques. The objective of this research was to apply environmental education strategies to reduce the use of agrochemicals in the Balsa en Medio community (Ecuador). Using Likert-type scales, a diagnosis was made of the level of knowledge and environmental behavior of the community members. Afterwards, the training plan was implemented applying environmental strategies on the preparation and application of compost, bioles, bioinsecticides and biopreparations with efficient microorganisms in seasonal crops. Finally, the final environmental knowledge and behavior of the participants was evaluated. The results of the diagnosis revealed that $100 \%$ of the participants presented a deficient environmental knowledge, while $98 \%$ of participants showed inappropriate environmental behavior. After applying an environmental education program, it was obtained that $21.15 \%$ of participants showed excellent environmental knowledge; $61.54 \%$ were in the good category and the remaining $17.31 \%$ revealed little knowledge; For environmental behavior, $44.23 \%$ of participants were adequate, in contrast, $55.77 \%$ showed inadequate environmental behavior. The workshops given in the community made it possible to significantly increase the level of environmental knowledge and behavior.
\end{abstract}

Keywords: Environmental education strategies; environmental knowledge; environmental behavior; rural community; Likert scale; training plan.

\footnotetext{
1 Escuela Superior Politécnica Agropecuaria de Manabí Manuel Félix López, Ecuador. 2 Universidad Nacional Mayor de San Marcos, Lima, Perú

a Autor para correspondencia: aaveiga@espam.edu.ec - ORCID: https://orcid.org/0000-0003-0603-6269

b E-mail: hugoc28@espam.edu.ec- ORCID: https://orcid.org/0000-0001-6413-4321

c E-mail: aaveiga@espam.edu.ec - ORCID: https://orcid.org/0000-0002-0301-0226

d E-mail: otinocog@unmsm.edu.pe - ORCID: https://orcid.org/0000-0002-7927-931X

e E-mail: falcantarab@unmsm.edu.pe - ORCID: https://orcid.org/0000-0001-9127-4450
} 


\section{INTRODUCCIÓN}

De acuerdo con la Declaración de Tbilisi, la educación ambiental debería mejorar una variedad de resultados para los participantes, incluyendo conciencia, conocimiento, actitudes, habilidades y comportamientos para abordar los problemas ambientales; hoy en día, estos resultados incluyen comportamientos de gestión que se extienden a lo cotidiano y continúan influyendo en los participantes después de recibir capacitaciones (O’Hare et al., 2020).

Además de varios resultados positivos medidos a través de cambios en las actitudes hacia el medio ambiente, la educación ambiental también es apreciada por su gran influencia en la formación de una visión global; debido a su carácter transdisciplinario, anima a las personas a establecer vínculos $\mathrm{y}$ asociaciones, $\mathrm{y}$ también tiene un efecto social importante, implicando una mayor interacción y comunicación entre las personas, teniendo también una fuerte dimensión ética (Ghiurcă, A., Lămăşanu, A., y Mihai, 2012). Para que la educación ambiental sea eficaz, no debe tratarse meramente como una disciplina científica, sellada herméticamente de otras disciplinas, sino integrada en todo el currículo escolar y en la vida cotidiana simultáneamente, permitiendo su reconocimiento en todas estas situaciones (Öllerer, 2015).

Resulta imperativo indicar que la educación es un área de investigación cada vez más relevante, debido a la emergente necesidad de educar a las personas para hacer frente a los problemas ambientales; requiriendo un nuevo sistema de aprendizaje que cambie los estilos de vida, transformando los principios ecológicos en hábitos (Gómez, E., y Carrillo, 2008); para ello, se aboga por la exploración de métodos de aprendizaje que permitan el desarrollo de competencias que ayuden a afrontar esta transición personal, yendo más allá del enfoque del conocimiento cognitivo (Gómez et al., 2020).

Sin embargo, la investigación en el área de la educación ambiental ha tendido a tener un alcance algo limitado; pues normalmente se centra en sitios únicos, como aulas, programas de organizaciones o estrategias en áreas naturales; si bien este trabajo es ciertamente importante, adoptar un enfoque tan estrecho ha significado que las relaciones entre la teoría y la práctica o entre diferentes sitios educativos a menudo no se hayan examinado; también ha significado que las formas en que las prácticas y perspectivas de la educación ambiental están profundamente arraigadas en contextos sociales, históricos y económicos particulares que no se han explorado por completo (Blum, 2012).

En tal sentido, las Escuelas de Campo (ECAs) constituyen un enfoque educativo que permite a los agricultores tomar decisiones adecuadas a la situación real del campo, basándose en su comprensión de los sistemas y procesos agroecológicos (van den Berg et al., 2021). Por tanto, esta estrategia permitió crear un entorno propicio para el aprendizaje en grupo, mediante el uso de ejercicios de campo (Lovera et al., 2016), análisis críticos y discusiones de grupo realizadas a intervalos regulares, permitiendo un enfoque centrado en el alumno para la educación de adultos y el marco para los dominios técnicos, prácticos y emancipadores del aprendizaje; con estos fundamentos educativos, se asegura un proceso de aprendizaje, acción y empoderamiento continuo por parte de sus participantes.

En la comunidad Balsa en Medio, la producción agrícola es predominante (Luque, 2016), y, la aplicación de agroquímicos se ha vuelto indispensable en cada sembrío; además, en esta zona, son escasas las intervenciones de educación ambiental que realmente permitan cambiar el modelo productivo. Por lo cual, el objetivo de la presente investigación fue aplicar estrategias de educación ambiental para la disminución del uso de agroquímicos en la comunidad Balsa en Medio, cantón Bolívar ManabíEcuador. El aporte práctico de este trabajo consiste en promover habilidades de producción ambientalmente amigables en comunidades rurales.

\section{MATERIALES Y MÉTODOS}

\section{1. Área de estudio}

La presente investigación se realizó en la comunidad Balsa en Medio en las coordenadas 617231; 9890816, perteneciente al cantón Bolívar, Manabí-Ecuador (Figura 1).

\subsection{Diagnóstico del nivel de conocimiento y comportamiento ambiental de los miembros de la Asociación Agropecuaria de la Comunidad Balsa en Medio}

Tomando en cuenta las características productivas de la comunidad en estudio, el diagnóstico se realizó mediante la valoración del conocimiento y comportamiento ambiental, a todos los miembros (52 personas) de la Asociación Agropecuaria de la Comunidad Balsa en MedioAGROBEM (Acuerdo Ministerial MAG - No. 010 - 12 01 - 2016), adaptando criterios establecidos por Barazarte, R., Neaman, A., Vallejo, F., García, (2014).

Para valorar oportunamente el comportamiento ambiental, se adaptaron categorías de la escala tipo Likert para examinar las percepciones y actitudes informadas de los encuestados; este enfoque de escala unidimensional está basado en la teoría de medición clásica, que utiliza un solo tipo de estímulo y un solo tipo de respuesta; donde los ítems de escala se generan a partir de operacionalizaciones o referentes empíricos de un constructo latente que a menudo es determinado por el investigador o el desarrollador de la escala; a cada categoría de respuesta se asignó un valor numérico (Ho, 2017).

Para el conocimiento ambiental, se aplicó un cuestionario de 10 preguntas de selección múltiple, donde cada interrogante tenía una respuesta correcta, calificándose con un valor de 1 , la elección incorrecta se puntuó con 0 , la nota final se obtuvo mediante sumatoria; misma que se categorizó en tres rangos i) malo para calificaciones $\leq 5$, ii) bueno entre 6 y 7 , y excelente a partir de 8 puntos (Cardona, J., Caro, A., González, J., y Franco, 2014).

Referente al comportamiento ambiental, se aplicó una encuesta de 10 ítems, en la cual se indicaba la frecuencia con la que los participantes realizaban determinadas acciones. En este caso, se eligieron tres opciones: nunca (puntuación $=0$ ), ii) a veces (puntuación $=0,5)$, y iii) 


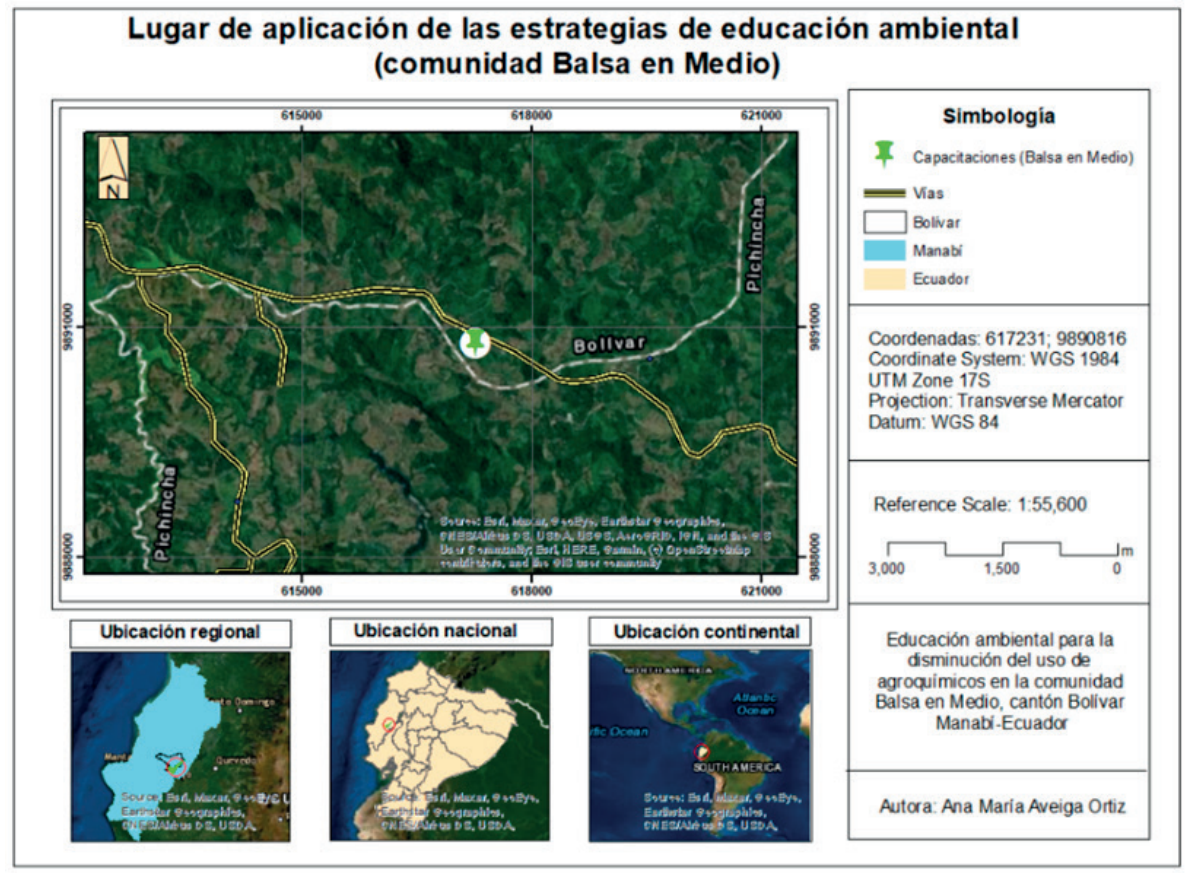

Figura 1. Ubicación geográfica donde se impartieron las capacitaciones (Balsa en Medio).

siempre (puntuación $=1$ ). La calificación final se obtuvo por sumatoria de los puntos obtenidos, se consideró un comportamiento ambiental adecuado si la nota final fue mayor o igual a 5 puntos, caso contrario, el comportamiento ambiental se consideró inadecuado.

En cuanto a la validación de las encuestas, se determinó la confiabilidad del instrumento en términos de Alpha de Cronbach, la cual implica el desarrollo y aplicación de pruebas, donde las mediciones de diversos aspectos se considera habitual; es decir, Alpha se emplea usualmente en la construcción de escalas utilizadas para medir actitudes y otros aspectos de carácter afectivo (Taber, 2018).

2.3. Plan de capacitación aplicando estrategias ambientales en la Comunidad Balsa en Medio

Se impartieron talleres teóricos a los miembros de Asociación Agropecuaria de la Comunidad Balsa en Medio; cada taller tuvo una duración estimada de tres horas, con la finalidad de orientar a los participantes en materia ambiental; abordando inconvenientes, soluciones y estrategias como: elaboración y aplicación de compost, bioles, bioinsecticidas y biopreparados con microorganismos eficientes en cultivos estacionales. La instrucción se aplicó mediante ilustraciones (descriptivas y expresivas), clases expositivas, videos, preguntas intercaladas, y analogías acordes a los temas en estudio. El esquema general de contenidos fue: generalizaciones, beneficios, técnicas de elaboración y aplicación.

Posteriormente, se llevó a cabo una etapa práctica mediante la metodología aprender haciendo misma que permite lograr interacción dinámica entre los participantes y el facilitador mediante métodos didácticos de aprendizaje. En la Figura 2 se detallan las actividades realizadas.

\subsection{Evaluación final del nivel de conocimiento y comportamiento ambiental de los miembros de la Asociación Agropecuaria de la Comunidad Balsa en Medio}

Después de realizar los cuatro talleres de capacitación, se evaluó el conocimiento y comportamiento ambiental final de los participantes mediante las técnicas descritas en la fase de diagnóstico. Así mismo, se realizó un análisis estadístico en el software SPSS versión 25.0; aplicando la prueba no paramétrica de Wilcoxon para comparar muestras relacionadas. Posteriormente, se evaluó la variación en el conocimiento y comportamiento ambiental y se analizó el efecto de las características sociodemográficas (edad, sexo, nivel de instrucción y estrato socioeconómico) mediante análisis de varianza de dos factores (ANOVA) (Baena \& Granero, 2014).

\section{RESULTADOS}

La fase de diagnóstico se realizó el 26 de noviembre de 2018 en la comunidad Balsa en Medio, contando con 52 participantes (AGROBEM), de los cuales 63,46\% pertenecen al género masculino y $36,64 \%$ al femenino. Los resultados del diagnóstico en cuanto a conocimiento ambiental se presentan en la Figura 1, obteniéndose que el $100 \%$ de los participantes presentaron conocimiento ambiental malo; mientras que, en lo referente a comportamiento ambiental, el $98 \%$ de participantes mostraron comportamiento ambiental inadecuado.

En base a este diagnóstico, se ejecutó el plan de capacitación, puesto que según criterios de Ghiurcă, A., Lămăşanu, A., y Mihai (2012), el grado de degradación de los factores ambientales está correlacionado con el grado de educación ambiental de la población rural; además, la 


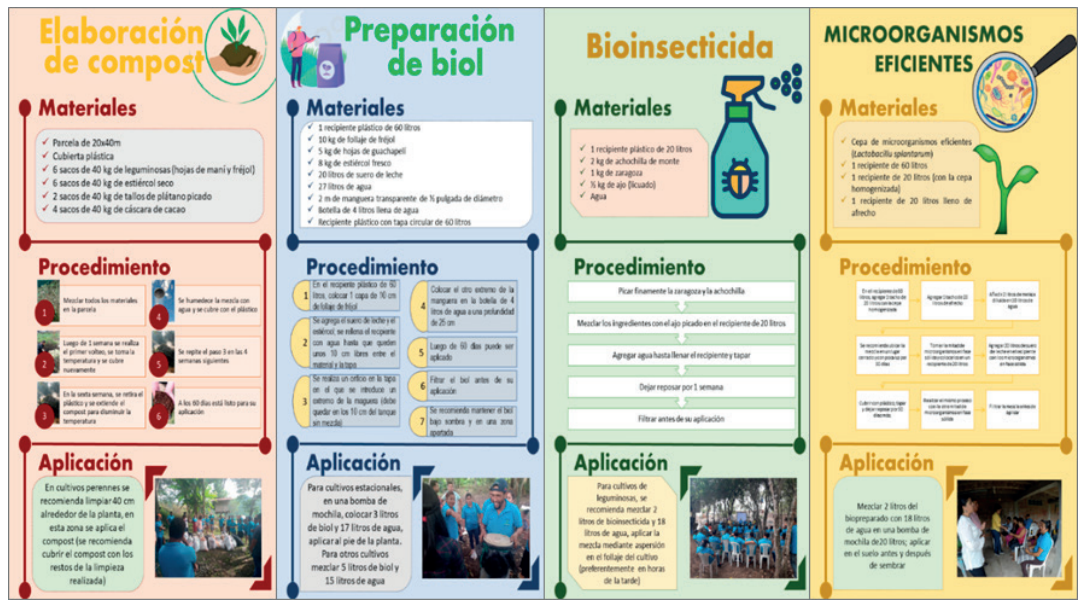

Figura 2. Detalle de las estrategias de educación ambiental aplicadas.

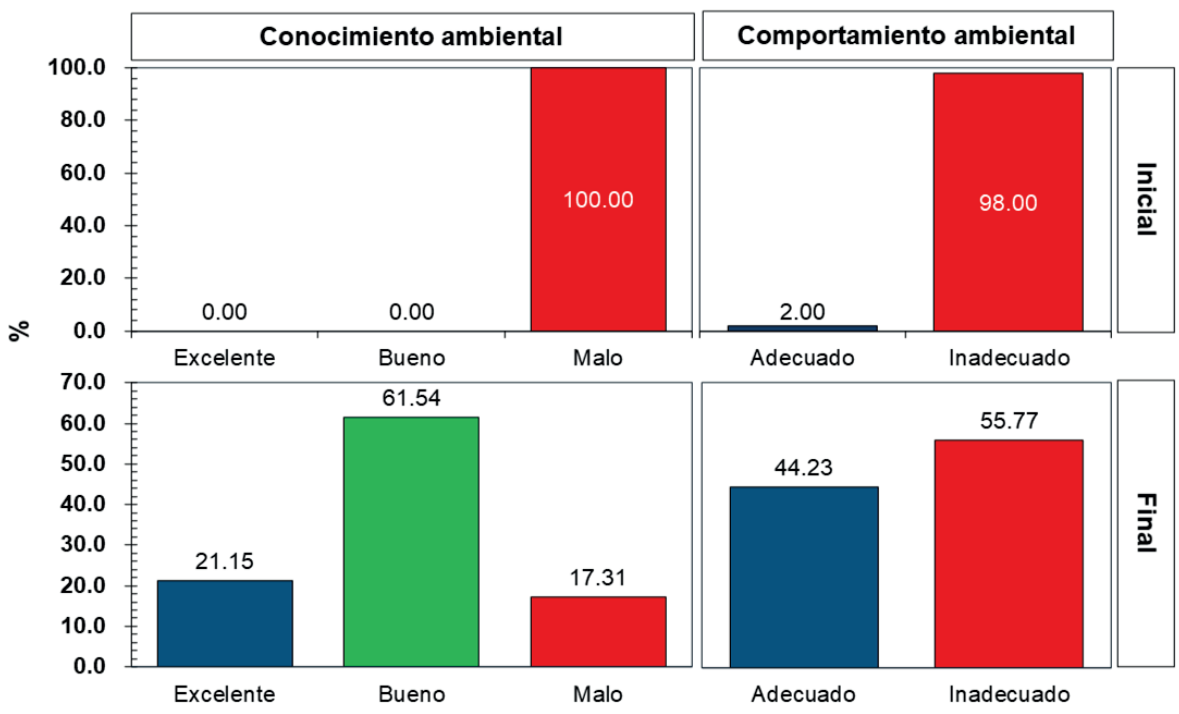

Figura 3. Contraste de la valoración del conocimiento y comportamiento ambiental de los miembros de AGROBEM.

situación económica, el desinterés de las autoridades locales por los temas ambientales y la falta de intervenciones educativas podrían condenar a las poblaciones rurales a empeorar sus condiciones de vida.

En cuanto a la ejecución de las cuatro estrategias ambientales (compost, biol, bioinsecticidas y microorganismos eficientes), se evidenció gran interés por parte de los miembros de AGROBEM en aplicar estas técnicas en sus cultivos. Una vez terminados los talleres, se realizó la valoración final del conocimiento y comportamiento ambiental el 29 de abril de 2019.

Del total de participantes, $69,23 \%$ pertenecen al estrato socioeconómico bajo y el $30,77 \%$ restante es del estrato medio. En cuanto al nivel de instrucción, el 78,85\% completó el nivel básico, mientras que el $21,15 \%$ el nivel medio. Referente a la edad, el 5,77\% es menor a 24 años, el $11,54 \%$ se encuentran en un rango de entre 25 y 34 años, el $34,62 \%$ pertenece al rango de 35 a 44 años, el 32,69\% está entre 45 y 54 años, el 11,54\% pertenece al rango entre 55 y 64 años, y existe un 3,85\% mayor o igual a 65 años; siendo un grupo diverso en edades.

La valoración final del conocimiento y comportamiento ambiental se muestra en la Figura 3; donde se evidencia que después de la intervención el $21,15 \%$ de participantes mostraron conocimiento ambiental excelente; $61.54 \%$ se ubicaron en la categoría bueno y el 17,31\% restante mostró escaso conocimiento. Para el comportamiento ambiental, el 44,23\% de participantes se ubicó en adecuado, en contrate, $55,77 \%$ aún mantiene comportamiento ambiental inadecuado.

Mediante el ANOVA aplicado, se determinó que la única variable que presenta un efecto sobre el conocimiento ambiental es el nivel de instrucción. Mientras que, para el comportamiento ambiental ninguna de las variables sociodemográficas es determinante (Tabla 1). 
Tabla 1. ANOVA aplicado a las características sociodemográficas de la población en estudio

\begin{tabular}{lllcc}
\hline ANOVA & & & F & Sig. \\
\hline Conocimiento & Sexo & & 1,049 & 0,311 \\
ambiental & Edad & Entre & 0,374 & 0,864 \\
& Nivel de instrucción & grupos & 6,166 & 0,016 \\
& Estrato socioeconómico & & 2,991 & 0,090 \\
Comportamiento & Sexo & & 0,736 & 0,395 \\
ambiental & Edad & Entre & 0,649 & 0,664 \\
& Nivel de instrucción & grupos & 0,109 & 0,742 \\
& Estrato socioeconómico & & 0,122 & 0,729 \\
\hline
\end{tabular}

${ }^{*}$ Valores significativos $(p<0,05)$

Antes de la intervención el valor máximo de conocimiento ambiental de los miembros de AGROBEM era de 5 , sin embargo, después la puntuación máxima fue de 9; mostrando un aumento considerable en este criterio (Figura 4). Además, al aplicar la prueba no paramétrica de Wilcoxon, con nivel de confianza 95\%, se obtuvo significancia estadística $(\mathrm{p}=0.000)$.

En cuanto al comportamiento ambiental (Figura 5), el máximo pasó de 6 a 10; y, los resultados de la prueba no paramétrica de Wilcoxon, con nivel de confianza 95\%, mostraron diferencia significativa $(\mathrm{p}=0,000)$. Resultados que se relacionan con los de Villadiego-Lorduy et al. (2015), quienes afirman que, en términos estadísticos, todos los participantes mejoraron sus actitudes, conocimientos y comportamiento ambientales.

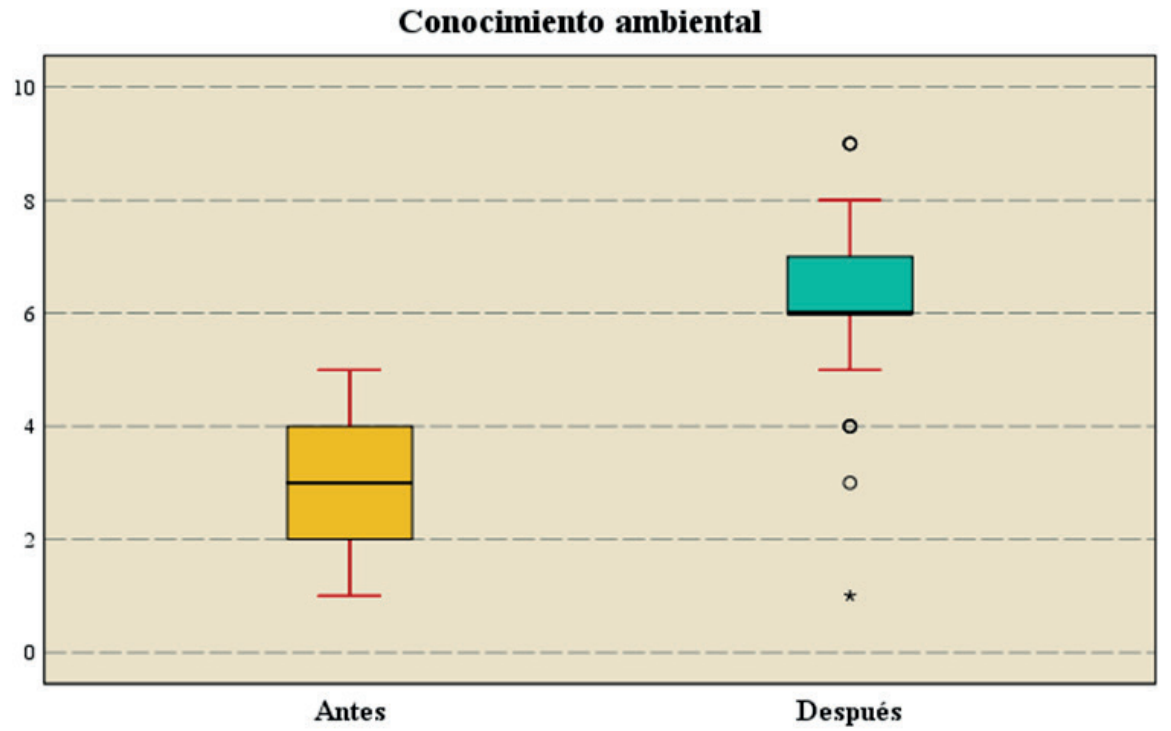

Figura 4. Variación del conocimiento ambiental en los miembros de AGROBEM.

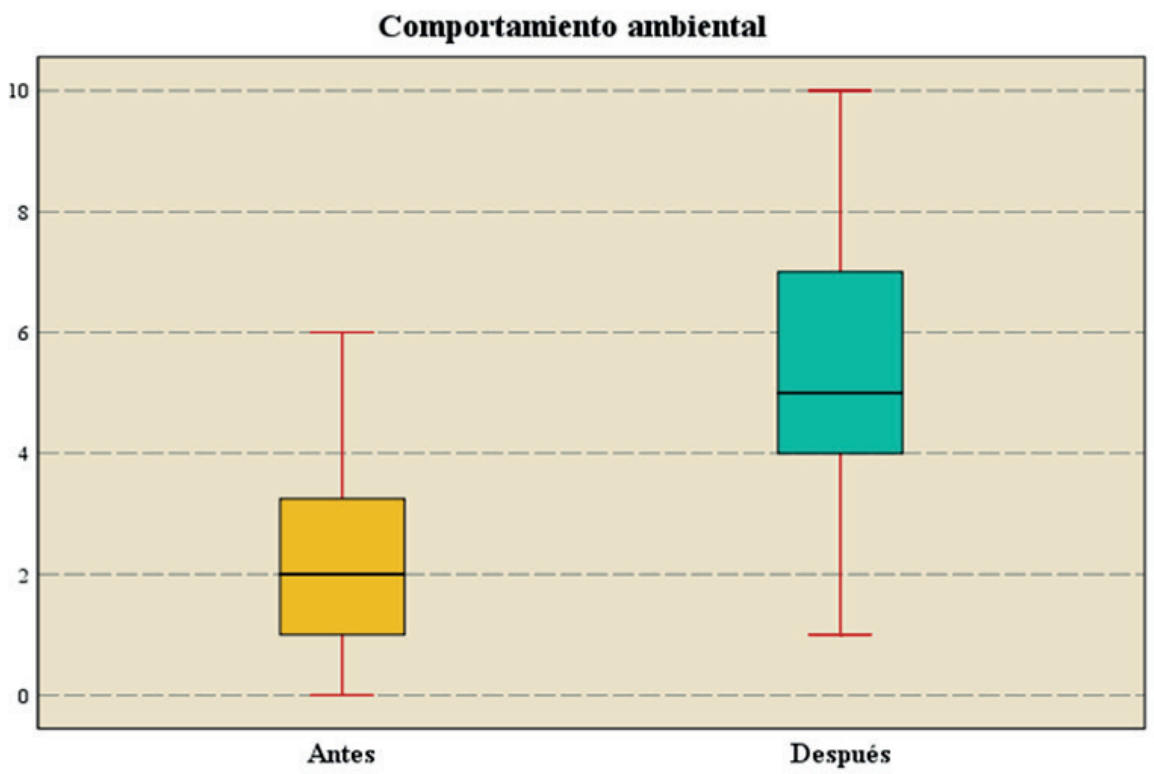

Figura 5. Variación del comportamiento ambiental en los miembros de AGROBEM. 
La fiabilidad de los datos obtenidos fue alta, con un coeficiente Alpha de Cronbach de 0,960 (Tabla 2).

Tabla 2. Análisis de fiabilidad de los datos obtenidos mediante las encuestas aplicadas

\begin{tabular}{cc}
\hline \multicolumn{2}{c}{ Estadísticas de fiabilidad } \\
\hline Alfa de Cronbach & N de elementos \\
, 960 & 4 \\
\hline
\end{tabular}

\section{DISCUSIÓN}

Ateniendo a los resultados obtenidos, en la comunidad Balsa en Medio se logró un cambio en el conocimiento y comportamiento ambiental. Ahora bien, el conocimiento ambiental no define el comportamiento ambiental, que de hecho corresponde a la ejecución de conductas proambientales (Balundè et al., 2019). Pues, a pesar de que se posea un profundo conocimiento ambiental, las prácticas efectivas del comportamiento ambiental parecen limitadas sobre todo para las poblaciones rurales donde la disponibilidad de información y capacitación son escasas (Côrtes et al., 2016). Por su parte, Wi \& Chang (2019) refieren que la comprensión ambiental de los individuos resulta de la transformación del conocimiento, mientras el comportamiento describe el desempeño dado (por ejemplo, agricultura ecológica) y conduce a un resultado medible (por ejemplo, certificaciones ambientales).

Asimismo, Villadiego-Lorduy et al. (2015) mencionan que la influencia de las características sociodemográficas sobre la conducta proambiental no es concluyente y podrían incluso brindar resultados contradictorios, indicando la compleja naturaleza de los hallazgos presentados. Por otra parte, Côrtes et al. (2016) identificaron una clara correlación entre el nivel de instrucción y el comportamiento ambiental; mientras que, en esta investigación, la misma variable mostró un efecto estadísticamente significativo sobre el conocimiento ambiental.

En cuanto a fiabilidad de los resultados, otros estudios sobre comportamientos y actitudes ambientales han dado como aceptables valores de Alpha de Cronbach de entre 0,6 y 0,7 indicando buena consistencia interna de los datos analizados (Biasutti \& Frate, 2017 ; Büyüktaşkapu Soydan \& ÖztürkSamur, 2017).

\section{CONCLUSIONES}

En el área de estudio se evidencia gran interés por la aplicación de técnicas de producción ambientalmente amigables; a pesar de las limitaciones de carácter financiero puesto que el $69,23 \%$ de participantes pertenecen al estrato socioeconómico bajo. Los talleres impartidos en la comunidad permitieron aumentar de manera significativa el nivel de conocimiento y comportamiento ambiental, ratificando la importancia de las intervenciones de este tipo en comunidades rurales. Así mismo, los hallazgos de esta investigación muestran que las estrategias de educación ambiental, aplicadas para disminuir el uso de agroquímicos en las actividades productivas de los miembros de la Asociación Agropecuaria de la Comunidad Balsa en
Medio (AGROBEM) tuvo un impacto positivo que pudiera contribuir a la sostenibilidad en comunidades rurales, aportando así a la reducción de la contaminación ambiental y asegurando la soberanía alimentaria.

\section{AGRADECIMIENTOS}

Los autores de este trabajo agradecen la valiosa colaboración de: la Asociación Agropecuaria de la Comunidad Balsa en Medio-AGROBEM, Ing. José Manuel Calderón Pincay, Ing. Carlos Luís Banchón Bajaña (docentes de la Carrera Ingeniería Ambiental de la ESPAM MFL), Ing. Fabián Fabricio Peñarrieta Macías (Técnico Investigador del Laboratorio de Química Ambiental y Suelos de la ESPAM MFL) y María Isabel Delgado Moreira.

\section{REFERENCIAS}

Baena, A., \& Granero, A. (2014). Estudio cuasi-experimental sobre actitudes de educación ambiental en educación física. Cultura, Ciencia y Deporte, 9(25), 25-33. https://doi. org/10.12800/ccd.v9i25.386

Balundè, A., Perlaviciute, G., \& Steg, L. (2019). The relationship between people's environmental considerations and pro-environmental behavior in Lithuania. Frontiers in Psychology, 10(OCT). https://doi.org/10.3389/ fpsyg.2019.02319

Barazarte, R., Neaman, A., Vallejo, F., García, P. (2014). El conocimiento ambiental y el comportamiento proambiental de los estudiantes de la Enseñanza media, en la Región de Valparaíso (Chile). Revista de Educacion, 364, 66-92. https://doi.org/10.4438/1988-592X-RE-2014-364-255

Biasutti, M., \& Frate, S. (2017). A validity and reliability study of the Attitudes toward Sustainable Development scale. Environmental Education Research, 23(2), 214-230. https:// doi.org/10.1080/13504622.2016.1146660

Blum, N. (2012). Education, community engagement and sustainable development: Negotiating environmental knowledge in Monteverde, Costa Rica. In Education, Community Engagement and Sustainable Development: Negotiating Environmental Knowledge in Monteverde, Costa Rica (Vol. 9789400725). https://doi.org/10.1007/97894-007-2527-0

Büyüktaşkapu Soydan, S., \& ÖztürkSamur, A. (2017). Validity and reliability study of environmental awareness and attitude scale for preschool children. International Electronic Journal of Environmental Education, 7(1), 78-97. https:// doi.org/10.18497/iejee-green.65615

Cardona, J., Caro, A., González, J., y Franco, S. (2014). Construcción y evaluación de una escala sobre conocimientos en primeros auxilios en estudiantes de educación media Medellín-Bello. CES Medicina, 28(1), 35-48. http://www. scielo.org.co/pdf/cesm/v28n1/v28n1a04.pdf

Côrtes, P. L., Dias, A. G., Fernandes, M. E. da S. T., \& Pamplona, J. M. V. (2016). Environmental behavior: A comparative study between Brazilian and Portuguese students. Ambiente e Sociedade, 19(3), 111-134. https://doi.org/10.1590/18094422ASOC139099V1932016 
Ghiurcă, A., Lămăşanu, A., y Mihai, F. (2012). Environmental education in rural areas - a real support for sustainable development. Revista Cientifica Europea, 117-122. https:// mpra.ub.uni-muenchen.de/61508/1/MPRA_paper_61508. pdf

Gómez, A. M., Valor, C., \& Carrero, I. (2020). Mindfulness in education for sustainable development to nurture socioemotional competencies: A systematic review and meta-analysis. Environmental Education Research, 26(11), 1527-1555. https://doi.org/10.1080/13504622.2020.177726

Gómez, E., y Carrillo, N. (2008). El paradigma de la educación ambiental y los conflictos sociales en minería. Revista Del Instituto de Investigación de La Facultad de Ingeniería Geológica, Minera, Metalurgica y Geográfica, 11(22), 8288. https://revistasinvestigacion.unmsm.edu.pe/index.php/ iigeo/article/view/474/405

Ho, G. W. K. (2017). Examining Perceptions and Attitudes: A Review of Likert-Type Scales Versus Q-Methodology. Western Journal of Nursing Research, 39(5), 674-689. https://doi.org/10.1177/0193945916661302

Lovera, D., Chillon, R., Huallpa, W., Quispe, N., Valencia, D., Marreros, G., Arana, K., Ordoño, D., Chilon, H., Sánchez, L., Mamani, M., Salcedo, G., Lovera, D. (2016). Diagnóstico socioambiental participativo en el centro poblado de Huarangal, distrito de Carabayllo, región Lima. Revista Del Instituto de Investigación de La Facultad de Ingeniería Geológica, Minera, Metalurgica y Geográfica, 18(35), 49-56. https://revistasinvestigacion.unmsm.edu.pe/ index.php/iigeo/article/view/11710/10500

Luque, J. (2016). El óxido nitroso y su influencia en el calentamiento global, provincia de Manabí, Ecuador Revista Del Instituto de Investigación de La Facultad de Ingeniería Geológica, Minera, Metalurgica y Geográfica, 18(35), 75-82. https://revistasinvestigacion.unmsm.edu.pe/ index.php/iigeo/article/view/11844/10571
O’Hare, A., Powell, R. B., Stern, M. J., \& Bowers, E. P. (2020). Influence of educator's emotional support behaviors on environmental education student outcomes. Environmental Education Research, 26(11), 1556-1577. https://doi.org/10. $1080 / 13504622.2020 .1800593$

Öllerer, K. (2015). Environmental education - the bumpy road from childhood foraging to literacy and active responsibility. Journal of Integrative Environmental Sciences, 12(3), 205216. https://doi.org/10.1080/1943815X.2015.1081952

Taber, K. S. (2018). The Use of Cronbach's Alpha When Developing and Reporting Research Instruments in Science Education. Research in Science Education, 48(6), 1273 1296. https://doi.org/10.1007/s11165-016-9602-2

van den Berg, H., Phillips, S., Poisot, A. S., Dicke, M., \& Fredrix, M. (2021). Leading issues in implementation of farmer field schools: a global survey. Journal of Agricultural Education and Extension, 27(3), 341-353. https://doi. org/10.1080/1389224X.2020.1858891

Villadiego-Lorduy, J., Huffman-Schwocho, D. P., GuerreroGómez, S. Y., Cortecero-Bossio, A., \& Rivero-Espitia, S. (2015). Factores de incidencia de conductas ambientales negativas hacia las ciénagas de Bañó y Los Negros. Revista Electrónica Educare, 19(3), 1-16. https://doi.org/10.15359/ ree.19-3.6

Wi, A., \& Chang, C. H. (2019). Promoting pro-environmental behaviour in a community in Singapore-from raising awareness to behavioural change. Environmental Education Research, 25(7), 1019-1037. https://doi.org/10.1080/13504 622.2018 .1528496 